\title{
MONOTONICITY AND CONCAVITY OF INTEGRAL FUNCTIONALS INVOLVING AREA MEASURES OF CONVEX BODIES
}

\author{
ANDREA COLESANTI, DANIEL HUG AND EUGENIA SAORÍN GÓMEZ
}

\begin{abstract}
For a broad class of integral functionals defined on the space of $n$-dimensional convex bodies, we establish necessary and sufficient conditions for monotonicity, and necessary conditions for the validity of a Brunn-Minkowski type inequality. In particular, we prove that a BrunnMinkowski type inequality implies monotonicity, and that a general Brunn-Minkowski type inequality is equivalent to the functional being a mixed volume.
\end{abstract}

\section{INTRODUCTION}

For a broad class of homogeneous functionals $\mathbf{F}$ defined on $\mathcal{K}^{n}$, the space of convex bodies (non-empty compact convex sets) in $\mathbb{R}^{n}$, a Brunn-Minkowski type inequality of the following form holds true,

$$
\mathbf{F}((1-t) K+t L)^{1 / \alpha} \geq(1-t) \mathbf{F}(K)^{1 / \alpha}+t \mathbf{F}(L)^{1 / \alpha}
$$

for all $K, L \in \mathcal{K}^{n}$ and $t \in[0,1]$, where $(1-t) K+t L$ is a Minkowski combination of $K$ and $L$, and $\alpha$ is the degree of homogeneity of $\mathbf{F}$. In other words, condition (1) states that $\mathbf{F}^{\mathbf{1 / \alpha}}$ is concave on $\mathcal{K}^{n}$. The archetype of these inequalities is the classical Brunn-Minkowski inequality, in which $\mathbf{F}$ is the $n$-dimensional volume functional (Lebesgue measure) and $\alpha=n$. This inequality is one of the cornerstones of convex geometry and connects this subject to many other areas of mathematics. The interested reader is referred to the survey paper [5] by Gardner. Other important examples come from the realm of convex geometry itself (intrinsic volumes, mixed volumes and many others) or from analysis (e.g., eigenvalues of elliptic operators, various notions of capacities); see for instance [2] and [11].

In many remarkable cases, a functional $\mathbf{F}$ which satisfies a Brunn-Minkowski type inequality is accompanied by other significant properties like continuity, additivity, and monotonicity with respect to set inclusion. One of the purposes of this paper is to investigate the interplay between a Brunn-Minkowski type inequality and monotonicity for some integral functionals involving area measures of convex bodies (see Section 2 for definitions and references). For a continuous function $f$ defined on the unit sphere $\mathbb{S}^{n-1}$ of $\mathbb{R}^{n}$ and an integer $i \in\{1, \ldots, n-1\}$, we define

$$
K \mapsto \mathbf{F}(K):=\int_{\mathbb{S}^{n-1}} f(u) S_{i}(K ; d u),
$$

Date: April 16, 2018.

2010 Mathematics Subject Classification. Primary: 52A20; Secondary: 26D15 49Q20 52A39 52A40.

Key words and phrases. Convex bodies; Brunn-Minkowski inequality; area measure; monotonic functional. 
where $S_{i}(K ; \cdot)$ denotes the $i$ th area measure of $K$. By the properties of area measures $([11$, Section 5.1]) and the continuity of $f$, the functional $\mathbf{F}$ is continuous with respect to the Hausdorff metric, translation invariant and homogeneous of degree $i$.

In the particular case where $f$ is the support function of some fixed convex body $L$, the functional $\mathbf{F}$ is in fact a mixed volume and has two additional interesting properties. The first is monotonicity with respect to set inclusion, which means that for all $K, L \in \mathcal{K}^{n}$,

$$
K \subset L \Rightarrow \mathbf{F}(K) \leq \mathbf{F}(L) .
$$

Second, F satisfies a Brunn-Minkowski type inequality (1) with $\alpha=i$, that is,

$$
\mathbf{F}((1-t) K+t L)^{1 / i} \geq(1-t) \mathbf{F}(K)^{1 / i}+t \mathbf{F}(L)^{1 / i}
$$

for all $K, L \in \mathcal{K}^{n}$ and $t \in[0,1]$. Since mixed volumes are non-negative, the $i$ th root is well defined.

For general $f$, other than a support function, we cannot expect $\mathbf{F}$ to satisfy either (3) or (4). Let us examine the case $i=n-1$. In [8], McMullen proved that, in this case, (3) implies that $f$ is a support function. A corresponding result for the Brunn-Minkowski inequality has been recently established in [3]. There it is shown that (4) implies that $f$ is a support function. Hence, for $i=n-1$, both (3) and (4) are equivalent to the fact that $\mathbf{F}$ is a mixed volume, and therefore they are equivalent to each other.

These equivalences are no longer true for $i<n-1$. For instance when $i=1$, the functional $\mathbf{F}$ is linear with respect to the Minkowski addition, and, in particular, it satisfies (4) for every choice of $f$. On the other hand, as we will see in Theorem 1.2, $\mathbf{F}$ is not monotonic for every $f$.

In the first part of this paper we find a condition on $f$ which is equivalent to monotonicity of $\mathbf{F}$. We first present this condition in the smooth case, that is, for $f \in C^{2}\left(\mathbb{S}^{n-1}\right)$. We need to introduce some notation. For $u \in \mathbb{S}^{n-1}$, we define the $(n-1) \times(n-1)$ matrix

$$
Q(f, u):=\left(f_{i j}(u)+f(u) \delta_{i j}\right)_{i, j=1}^{n-1},
$$

where $f_{i j}$ are the second covariant derivatives of $f$ with respect to an orthonormal frame on $\mathbb{S}^{n-1}$ and $\delta_{i j}$ are the usual Kronecker symbols. Hence, $Q(f, u)$ is the spherical Hessian matrix of $f$ at $u$ plus $f(u)$ times the identity matrix (see Section 2 again for details). This is a symmetric matrix, and we will denote by $\lambda_{i}(u), i=1, \ldots, n-1$, its eigenvalues. Note that if $\bar{f}$ denotes the 1-homogeneous extension of $f$ to $\mathbb{R}^{n}$ and $x \in \mathbb{R}^{n} \backslash\{0\}$, then the set of eigenvalues of the Euclidean Hessian matrix of $\bar{f}$ at $x$, denoted by $D^{2} \bar{f}(x)$, is $\left\{\lambda_{1}(u), \ldots, \lambda_{n-1}(u), 0\right\}$, where $u=$ $x /\|x\| \in \mathbb{S}^{n-1}$. In particular, the convexity of $\bar{f}$ is equivalent to the fact that $Q(f, u)$ is positive semi-definite for every $u$ (see [3, Appendix]).

To state our main results we need the following definition.

Definition 1.1. Let $f \in C^{2}\left(\mathbb{S}^{n-1}\right)$ and $i \in\{1, \ldots, n-1\}$. We say that $f$ satisfies condition $(\mathbf{M})_{i}$ if for every $u \in \mathbb{S}^{n-1}$ and $I \subset\{1, \ldots, n-1\}$ with $|I|=n-i$, we have

$$
\sum_{i \in I} \lambda_{i}(u) \geq 0
$$

where $|I|$ denotes the cardinality of $I$. 
In other words, for any choice of $(n-i)$ eigenvalues of $Q(f, u)$, their sum is non-negative. Note that if $f$ satisfies $(\mathbf{M})_{i}$, for some $i$, then it also satisfies $(\mathbf{M})_{j}$ for every $j \leq i$. The following result asserts that condition $(\mathbf{M})_{i}$ is equivalent to monotonicity of $\mathbf{F}$.

Theorem 1.2. Let $f \in C^{2}\left(\mathbb{S}^{n-1}\right)$ and $i \in\{1, \ldots, n-1\}$. Then the functional $\mathbf{F}$ defined by (2) satisfies (3) if and only if $f$ satisfies condition $(\mathbf{M})_{i}$.

In the case $i=n-1$ condition $(\mathbf{M})_{i}$ amounts to the fact that each eigenvalue must be non-negative, that is, $Q(f, u)$ is positive semi-definite everywhere on $\mathbb{S}^{n-1}$, and then the 1homogeneous extension $\bar{f}$ of $f$ is convex. But this in turn is equivalent to saying that $f$ is a support function. Hence we have an alternative proof of the result of McMullen [8], at least in the smooth case, but our procedure extends to the general case $f \in C\left(\mathbb{S}^{n-1}\right)$, as the Theorem 1.4 shows.

In the other limiting case, $i=1$, condition (5) means that the trace of $Q(f, u)$ is non-negative for every $u$; equivalently,

$$
\operatorname{trace}\left(D^{2} \bar{f}(x)\right)=\Delta \bar{f}(x) \geq 0 \quad \text { for all } x \neq 0,
$$

where $\Delta$ denotes the Euclidean Laplace operator, i.e., $\bar{f}$ is a subharmonic function in $\mathbb{R}^{n} \backslash\{0\}$.

In general, condition (5) is related to the so-called $r$-convexity of $f$ or, more precisely, of its 1-homogeneous extension. We recall that a function $g \in C^{2}(\Omega)$, where $\Omega$ is an open subset of $\mathbb{R}^{n}$, is said to be $k$-convex, for some $k \in\{1, \ldots n\}$, if for every $x \in \Omega$ and for $j=1, \ldots, k$ the $j$ th elementary symmetric function of the eigenvalues of $D^{2} g(x)$ is non-negative. In particular, it can be seen that $n$-convexity is equivalent to the usual convexity. It is known (see for instance [10], Prop. 1.3.3) that if $g$ is $k$-convex, then, for every $x \in \Omega$ and for every choice of $n-k+1$ distinct eigenvalues of $D^{2} g(x)$, their sum is non-negative. Hence we have the following corollary.

Corollary 1.3. Let $i \in\{1, \ldots, n-1\}$. Let $f \in C^{2}\left(\mathbb{S}^{n-1}\right)$, and let $\bar{f}$ be its 1-homogeneous extension. If $\bar{f}$ is $i$-convex in $\mathbb{R}^{n} \backslash\{0\}$, then the functional defined by (2) is monotonic.

Theorem 1.2 is complemented by the following statement concerning the case in which $f$ is just continuous.

Theorem 1.4. Let $f \in C\left(\mathbb{S}^{n-1}\right)$ and let $i \in\{1, \ldots, n-1\}$. Then the functional $\mathbf{F}$ defined by (2) satisfies (3), i.e., it is monotonic w.r.t. set inclusion, if and only if there exists a sequence $f_{k} \in C^{2}\left(\mathbb{S}^{n-1}\right), k \in \mathbb{N}$, converging to $f$ uniformly on $\mathbb{S}^{n-1}$ and such that $f_{k}$ satisfies condition $(\mathbf{M})_{i}$ for every $k \in \mathbb{N}$.

In Section 4, we consider the case in which $\mathbf{F}$ satisfies a Brunn-Minkowski type inequality and prove the following theorem.

Theorem 1.5. Let $i \in\{2, \ldots, n-1\}$ and let $f \in C^{2}\left(\mathbb{S}^{n-1}\right)$ be such that the functional $\mathbf{F}$ defined by (2) is non-negative and satisfies the Brunn-Minkowski type inequality (4). Then $f$ satisfies condition $(\mathbf{M})_{i}$.

Theorem 1.5 provides a necessary condition on $f$ so that $\mathbf{F}$ satisfies (4). However we do not know whether this condition is sufficient as well, apart from the case $i=n-1$ in which the answer is affirmative, as proved in [3]. Theorem 1.5 has the following corollary. 
Corollary 1.6. Let $i \in\{2, \ldots, n-1\}$ and let $f \in C^{2}\left(\mathbb{S}^{n-1}\right)$ be such that the functional $\mathbf{F}$ defined by (2) is non-negative and satisfies the Brunn-Minkowski inequality (4). Then $\mathbf{F}$ is monotonic.

In the case where $f$ is an even function in the sense that $f(-u)=f(u)$ for every $u \in \mathbb{S}^{n-1}$, and continuous, we have the following extension of Theorem 1.5 and Corollary 1.6 (in the spirit of Theorem 1.4).

Theorem 1.7. Let $i \in\{2, \ldots, n-1\}$, let $f \in C\left(\mathbb{S}^{n-1}\right)$ be even, and let $\mathbf{F}$ be defined as in (2). If $\mathbf{F}$ is non-negative and satisfies inequality (4), then there exists a sequence of functions $f_{k} \in C^{2}\left(\mathbb{S}^{n-1}\right), k \in \mathbb{N}$, which converges uniformly to $f$ on $\mathbb{S}^{n-1}$ such that $f_{k}$ satisfies condition $(\mathbf{M})_{i}$ for every $k \in \mathbb{N}$. In particular, $\mathbf{F}$ is monotonic.

In Section 4 we will see that the previous result also holds when the symmetry assumption on $f$ is replaced by the existence of second weak derivatives in the sense of Sobolev spaces.

Functionals defined by means of (2) can be seen as examples of more general integral functionals. Given $K_{1}, \ldots, K_{n-1} \in \mathcal{K}^{n}$, let $S\left(K_{1}, \ldots, K_{n-1} ; \cdot\right)$ be their mixed area measure (see Section 2 for precise definitions). If $f \in C\left(\mathbb{S}^{n-1}\right)$ and $i \in\{1, \ldots, n-1\}$, let the functional $\mathbf{F}: \mathcal{K}^{n} \longrightarrow \mathbb{R}$ be defined by

$$
\mathbf{F}(K)=\int_{S^{n-1}} f(u) S\left(K[i], K_{1}, \ldots, K_{n-i-1} ; d u\right) .
$$

The functional in (2) is recovered from (6) in the special case where $K_{1}, \ldots, K_{n-i-1}$ coincide with the Euclidean unit ball. If $f$ is the support function of some convex body $L$, then $\mathbf{F}$ equals the mixed volume

$$
V\left(L, K[i], K_{1}, \ldots, K_{n-i-1}\right) .
$$

In this case, $\mathbf{F}$ satisfies a Brunn-Minkowski type inequality for any choice of $L, K_{1}, \ldots, K_{n-i-1} \in$ $\mathcal{K}^{n}$. This result is called general Brunn-Minkowski inequality (see [11, Theorem 6.4.3]). In the last section of this paper we prove that this property characterizes support functions.

Theorem 1.8. Let $f \in C\left(S^{n-1}\right)$ and $i \in\{2, \ldots, n-1\}$ be such that for any choice of convex bodies $K_{1}, \ldots, K_{n-i-1} \in \mathcal{K}^{n}$ the functional $\mathbf{F}: \mathcal{K}^{n} \longrightarrow \mathbb{R}$ defined by (6) is non-negative and satisfies

$$
\mathbf{F}((1-t) K+t L)^{1 / i} \geq(1-t) \mathbf{F}(K)^{1 / i}+t \mathbf{F}(L)^{1 / i}
$$

for all $t \in[0,1]$ and $K, L \in \mathcal{K}^{n}$. Then $f$ is the support function of a convex body.

The general Brunn-Minkowski inequality (7) for the functional $\mathbf{F}$ defined as in (6) implies that

$$
\mathbf{F}((1-t) K+t L) \geq \min \{\mathbf{F}(K), \mathbf{F}(L)\}
$$

for all $K, L \in \mathcal{K}^{n}$ and $t \in[0,1]$, which is in general weaker than (7). However, in many cases it can be shown to be equivalent to it by a standard argument based on homogeneity. Note that (8) does not require $\mathbf{F}$ to be non-negative a priori.

The characterization theorem proved in [3] for the functional $\mathbf{F}$ defined by (2) in the case $i=n-1$ was proved under the assumption (8). This leads to the following extension of Theorem 1.8 in which condition (7) of Theorem 1.8 is replaced by (8) and the requirement that $\mathbf{F}$ be nonnegative is removed. 
Theorem 1.9. Let $f \in C\left(S^{n-1}\right)$ and $i \in\{2, \ldots, n-1\}$ be such that for any choice of convex bodies $K_{1}, \ldots, K_{n-i-1} \in \mathcal{K}^{n}$ the functional $\mathbf{F}: \mathcal{K}^{n} \longrightarrow \mathbb{R}$ defined by (6) satisfies (8). Then $f$ is the support function of a convex body.

\section{PRELIMINARIES}

We work in the $n$-dimensional Euclidean space $\mathbb{R}^{n}, n \geq 2$, endowed with the usual scalar product $\langle\cdot, \cdot\rangle$ and norm $\|\cdot\|$. We denote by $B^{n}$ the closed unit ball centered at the origin, and by $\mathbb{S}^{n-1}$ the unit sphere. Throughout the paper we will often use the convention that we sum over repeated indices.

2.1. Convex bodies. As stated in the introduction, for $n \geq 1$ we denote by $\mathcal{K}^{n}$ the collection of all non-empty compact convex subsets of $\mathbb{R}^{n}$, which are called convex bodies, for short. Our reference text on the theory of convex bodies is the monograph [11] by Schneider. Given $K, L \in \mathcal{K}^{n}$ and $\alpha, \beta \geq 0$, we write $\alpha K+\beta L=\{\alpha x+\beta y \mid x \in K, y \in L\}$ for the Minkowski combination of $K$ and $L$ with coefficients $\alpha$ and $\beta$.

For $K \in \mathcal{K}^{n}$ we denote by $h_{K}$ the support function of $K$, considered as a function on the unit sphere. We recall that support functions behave linearly with respect to the operations introduced above. For $K, L \in \mathcal{K}^{n}$ and $\alpha, \beta \geq 0$, we have $h_{\alpha K+\beta L}=\alpha h_{K}+\beta h_{L}$. Another property of convex bodies which can be expressed in a simple way via support functions is set inclusion. Indeed, for $K, L \in \mathcal{K}^{n}$,

$$
K \subset L \quad \text { if and only if } \quad h_{K} \leq h_{L} \text { on } \mathbb{S}^{n-1} .
$$

We will frequently need to work with convex bodies whose boundary is smooth. Let us introduce the following notation. We say that a convex body $K$ with non-empty interior is of class $C_{+}^{2}$ (briefly, $K \in C_{+}^{2}$ ), if its boundary is of class $C^{2}$ and the Gauss curvature is strictly positive at every boundary point $x \in \partial K$.

For $\phi \in C^{2}\left(\mathbb{S}^{n-1}\right), u \in \mathbb{S}^{n-1}$, and $i, j \in\{1, \ldots, n-1\}$, we put

$$
q_{i j}(\phi, u):=\phi_{i j}(u)+\delta_{i j} \phi(u),
$$

where $\phi_{i j}$ denote the second covariant derivatives of $\phi$, computed with respect to a local orthonormal frame (of vector fields) on $\mathbb{S}^{n-1}$ and $\delta_{i j}$ denote the usual Kronecker symbols. Moreover we set

$$
Q(\phi, u)=\left(q_{i j}(\phi, u)\right)_{i, j=1}^{n-1} .
$$

All relevant quantities and conditions will be independent of the particular choice of a local orthonormal frame in the following. For the sake of brevity, we sometimes omit the variable $u$ and simply write $q_{i j}(\phi)$ or $Q(\phi)$. Note that the matrix $Q(\phi, u)$ is symmetric for every $\phi \in C^{2}\left(\mathbb{S}^{n-1}\right)$ and every $u \in \mathbb{S}^{n-1}$ (see [3, Section 2] for further details). In the special case when $\phi$ is a support function, the matrix $Q(\phi, \cdot)$ will play a crucial role in the sequel.

We set

$$
\mathfrak{C}=\left\{h \in C^{2}\left(\mathbb{S}^{n-1}\right) \mid Q(h, u)>0 \text { for all } u \in \mathbb{S}^{n-1}\right\},
$$

where the notation $A>0$ stands for the matrix $A$ being positive definite.

A proof of the following result can be deduced from [11, Sect. 2.5]. 
Proposition 2.1. If $K \in C_{+}^{2}$, then $h_{K} \in \mathfrak{C}$. Conversely, if $h \in \mathfrak{C}$, then there exists a uniquely determined $K \in C_{+}^{2}$ such that $h=h_{K}$.

The mixed volume of the convex bodies $K_{1}, \ldots, K_{n} \in \mathcal{K}^{n}$ is denoted by $V\left(K_{1}, \ldots, K_{n}\right)$. For the mixed area measure of $K_{1}, \ldots, K_{n-1} \in \mathcal{K}^{n}$, we write $S\left(K_{1}, \ldots, K_{n-1} ; \cdot\right)$; see [11, Chapter 5] for the definitions. If in one of these functionals a convex body $K$ is repeated $i$ times, we use the notation $K[i]$, for instance, we put

$$
V\left(K[i], K_{i+1}, \ldots, K_{n}\right):=V(\underbrace{K, \ldots, K}_{i \text {-imes }}, K_{i+1}, \ldots, K_{n}) .
$$

The mixed are measures are Borel measures defined on $\mathbb{S}^{n-1}$. For the properties of area measures we refer to [11, Section 5.1]. The close connection between mixed volumes and mixed area measures is expressed by the relation

$$
V\left(K_{1}, \ldots, K_{n}\right)=\frac{1}{n} \int_{\mathbb{S}^{n-1}} h_{K_{n}}(u) S\left(K_{1}, \ldots, K_{n-1} ; d u\right) .
$$

For a given a convex body $K$ and $i \in\{1, \ldots, n-1\}$, the $i$ th area measure of $K$ is denoted by $S_{i}(K, \cdot)$ and equals the special mixed area measure $S\left(K[i], B^{n}[n-i-1] ; \cdot\right)$.

For the proof of our main results it will be important to express the density of the area measures of a convex body $K$ in terms of the matrix $Q\left(h_{K}\right)$. Before stating such representations we need to recall some facts about elementary symmetric functions.

2.2. Elementary symmetric functions and densities of area measures. Let $N$ be an integer. We denote by $\operatorname{Sym}(N)$ the set of $N \times N$ symmetric matrices (with real entries). For an element $A \in \operatorname{Sym}(N)$ we write $A>0$ and $A \geq 0$ if $A$ is positive definite and positive semi-definite, respectively.

Let $A=\left(a_{j k}\right)_{j, k=1}^{N} \in \operatorname{Sym}(N)$, with eigenvalues $\lambda_{j}, j=1, \ldots, N$, and let $i \in\{0,1, \ldots, N\}$. We define $S_{i}(A)$ as the $i$ th elementary symmetric function of the eigenvalues of $A$, that is,

$$
S_{i}(A)=\sum_{1 \leq j_{1}<\cdots<j_{i} \leq N} \lambda_{j_{1}} \cdots \lambda_{j_{i}} \quad \text { if } i \geq 1,
$$

and $S_{0}(A)=1$. Note, in particular, that $S_{1}(A)$ and $S_{N}(A)$ are the trace and the determinant of $A$, respectively. An explicit description of $S_{i}(A)$ in terms of (the entries of) $A$ is provided in (12) below.

For $N, A$ and $i$ as above, and for $j, k \in\{1, \ldots, N\}$, we set

$$
S_{i}^{j k}(A)=\frac{\partial S_{i}}{\partial a_{j k}}(A)
$$

The $N \times N$ matrix consisting of the entries $S_{i}^{j k}(A)$ is sometimes called the ith cofactor matrix of $A$. We will also need the second derivatives of $S_{i}(A)$ with respect to the entries of $A$, which are denoted by

for every $i, j, r, s \in\{1, \ldots, N\}$.

$$
S_{i}^{j k, r s}(A):=\frac{\partial^{2} S_{i}}{\partial a_{j k} \partial a_{r s}}(A),
$$


Let $K$ be a convex body of class $C_{+}^{2}$ and $h \in \mathfrak{C}$ its support function. For $i \in\{1, \ldots, n-1\}$, the $i$ th area measure $S_{i}(K ; \cdot)$ of $K$ is absolutely continuous with respect to the Haussdorf measure $\mathcal{H}^{n-1}$ restricted to $\mathbb{S}^{n-1}$, and its density is given by the function

$$
u \mapsto S_{i}(Q(h), u), \quad u \in \mathbb{S}^{n-1},
$$

(see, for example, [11, 5.3.2] for a proof). In other words, for every $f \in C\left(\mathbb{S}^{n-1}\right)$ we have

$$
\mathbf{F}(K)=\int_{\mathbb{S}^{n-1}} f(u) S_{i}(Q(h, u)) \mathcal{H}^{n-1}(d u) .
$$

2.3. A lemma of Cheng and Yau. For $\phi \in C^{2}\left(\mathbb{S}^{n-1}\right)$ and $i \in\{1, \ldots, n-1\}$, consider the matrix

$$
\left(S_{i}^{j k}(Q(\phi, u))\right)_{j, k=1}^{n-1}
$$

as a function of $u \in \mathbb{S}^{n-1}$. The following lemma will be of great importance in the rest of this paper. It asserts that if we consider any of the columns of (11) as a vector field on $\mathbb{S}^{n-1}$, its divergence vanishes pointwise. The case $k=n-1$ was originally proved by Cheng and Yau in [1].

Lemma 2.2. Let $\phi \in C^{3}\left(\mathbb{S}^{n-1}\right)$ and $i \in\{1, \ldots, n-1\}$. Then, for every $k \in\{1, \ldots, n-1\}$,

$$
\sum_{j=1}^{n-1}\left(S_{i}^{j k}(Q(\phi, u))\right)_{j}=0 \quad \text { for all } u \in \mathbb{S}^{n-1}
$$

We will also need a further generalization of Lemma 2.2. Let $\phi, \psi \in C^{3}\left(\mathbb{S}^{n-1}\right)$. Then, for $u \in \mathbb{S}^{n-1}$, we define the matrix $M=M(u)=\left(m_{j k}(u)\right)_{j, k=1, \ldots, n-1}$ by

$$
m_{j k}(u)=S_{i}^{j k, r s}(Q(\phi, u)) q_{r s}(\psi, u)
$$

(remember that we use the summation convention).

Lemma 2.3. In the above notation, for every $k \in\{1, \ldots, n-1\}$,

$$
\sum_{j=1}^{n-1}\left(m_{j k}(u)\right)_{j}=0 \quad \forall u \in \mathbb{S}^{n-1} .
$$

Proof. The proof follows the argument used in the proof of [4, Lemma 1]. We use an explicit formula for the $i$ th cofactor matrix in terms of the entries of the original matrix (see for instance [9] or [10]). For $A \in \operatorname{Sym}(n-1)$ we have

$$
S_{i}(A)=\frac{1}{i !} \sum \delta\left(\begin{array}{c}
j_{1}, \ldots, j_{i} \\
k_{1}, \ldots, k_{i}
\end{array}\right) a_{j_{1} k_{1}} \cdots a_{j_{i} k_{i}}
$$

where the sum is taken over all possible indices $j_{s}, k_{s} \in\{1, \ldots, n-1\}$ (for $s=1, \ldots, i$ ) and the Kronecker symbol $\delta\left(\begin{array}{l}j_{1}, \ldots, j_{i} \\ k_{1}, \ldots, k_{i}\end{array}\right)$ equals 1 (respectively, -1 ) when $j_{1}, \ldots, j_{i}$ are distinct and 
$\left(k_{1}, \ldots, k_{i}\right)$ is an even (respectively, odd) permutation of $\left(j_{1}, \ldots, j_{i}\right)$; otherwise it is 0 . Using the above equality, we have, for every $j, k, r, s \in\{1, \ldots, n-1\}$,

$$
\begin{aligned}
S_{i}^{j k}(A) & =\frac{1}{(i-1) !} \sum \delta\left(\begin{array}{c}
j, j_{1}, \ldots, j_{i-1} \\
k, j_{1}, \ldots, k_{i-1}
\end{array}\right) a_{j_{1} k_{1}} \cdots a_{j_{i-1} k_{i-1}}, \\
S_{i}^{j k, r s}(A) & =\frac{1}{(i-2) !} \sum \delta\left(\begin{array}{c}
r, j, j_{1}, \ldots, j_{i-2} \\
s, k, k_{1}, \ldots, k_{i-2}
\end{array}\right) a_{j_{1} k_{1}} \cdots a_{j_{i-2} k_{i-2}} .
\end{aligned}
$$

For simplicity, in the following formulas we omit the variable $u \in \mathbb{S}^{n-1}$. Then for the matrix $m_{j k}$ we obtain

$$
m_{j k}=\frac{1}{(i-2) !} \sum_{r, s} \sum \delta\left(\begin{array}{c}
r, j, j_{1}, \ldots, j_{i-2} \\
s, k, k_{1}, \ldots, k_{i-2}
\end{array}\right) q_{j_{1} k_{1}}(\phi) \cdots q_{j_{i-2} k_{i-2}}(\phi) q_{r s}(\psi) .
$$

Hence

$$
\begin{aligned}
& (i-2) ! \sum_{j=1}^{n-1}\left(m_{j k}\right)_{j}= \\
& =\sum_{j=1}^{n-1} \sum_{r, s} \sum\left\{\delta\left(\begin{array}{c}
r, j, j_{1}, \ldots, j_{i-2} \\
s, k, k_{1}, \ldots, k_{i-2}
\end{array}\right) \times\right. \\
& \times\left[\left(\phi_{j_{1} k_{1} j}+\phi_{j} \delta_{j_{1} k_{1}}\right)\left(\phi_{j_{2} k_{2}}+\phi \delta_{i_{2} j_{2}}\right) \cdots\left(\phi_{j_{i-2} k_{i-2}}+\phi \delta_{j_{i-2} k_{i-2}}\right)+\cdots\right. \\
& \left.+\left(\phi_{j_{1} k_{1}}+\phi \delta_{j_{1} k_{1}}\right) \cdots\left(\phi_{j_{i-3} k_{i-3}}+\phi \delta_{j_{i-3} k_{i-3}}\right)\left(\phi_{j_{i-2} k_{i-2} j}+\phi_{j} \delta_{j_{i-2} k_{i-2}}\right)\right]\left(\psi_{r s}+\delta_{r s} \psi\right)+ \\
& \left.+\left(\phi_{j_{1} k_{1}}+\phi \delta_{j_{1} k_{1}}\right) \cdots\left(\phi_{j_{i-2} j_{i-2}}+\phi \delta_{j_{i-2} k_{i-2}}\right)\left(\psi_{r s j}+\delta_{r s} \psi_{j}\right)\right\}
\end{aligned}
$$

In the last sum, for fixed $j_{1}, \ldots, j_{i-2}, k_{1}, \ldots, k_{i-2}, j, r, s$, we split the terms into two types: those in which there are no third covariant derivatives of $\psi$, and those where a third derivative of $\psi$ appears. As for the first type, consider the terms

$$
A=\delta_{1}\left(\phi_{j_{1} k_{1} j}+\phi_{j} \delta_{j_{1} k_{1}}\right) C \quad \text { and } \quad B=\delta_{2}\left(\phi_{j k_{1} j_{1}}+\phi_{j_{1}} \delta_{j k_{1}}\right) C
$$

where

$$
\delta_{1}=\delta\left(\begin{array}{c}
r, j, j_{1}, j_{2}, \ldots, j_{i-2} \\
s, k, k_{1}, k_{2}, \ldots, k_{i-2}
\end{array}\right), \quad \delta_{2}=\delta\left(\begin{array}{c}
r, j_{1}, j, j_{2}, \ldots, j_{i-2} \\
s, k, k_{1}, k_{2}, \ldots, k_{i-2}
\end{array}\right)
$$

and

$$
C=\left(\phi_{j_{2} k_{2}}+\phi \delta_{j_{2} k_{2}}\right) \cdots\left(\phi_{j_{i-2} k_{i-2}}+\phi \delta_{j_{i-2} k_{i-2}}\right)\left(\psi_{r s}+\delta_{r s} \psi\right)
$$

Clearly $\delta_{2}=-\delta_{1}$. On the other hand, the third order covariant derivatives of a function $g \in$ $C^{3}\left(\mathbb{S}^{n-1}\right)$ satisfy the symmetry relations

$$
g_{\alpha \beta \gamma}=g_{\beta \alpha \gamma}, \quad \alpha, \beta, \gamma=1, \ldots, n-1,
$$

and

$$
g_{\alpha \beta \gamma}+g_{\gamma} \delta_{\alpha \beta} \equiv g_{\alpha \gamma \beta}+g_{\beta} \delta_{\alpha \gamma}, \quad \alpha, \beta, \gamma=1, \ldots, n-1
$$


Consequently,

$$
\begin{aligned}
A+B & =\delta_{1} C\left(\phi_{j_{1} k_{1} j}+\phi_{j} \delta_{j_{1} k_{1}}-\phi_{j k_{1} j_{1}}-\phi_{j_{1}} \delta_{j k_{1}}\right) \\
& =\delta_{1} C\left(\phi_{k_{1} j_{1} j}+\phi_{j} \delta_{j_{1} k_{1}}-\phi_{j k_{1} j_{1}}-\phi_{j_{1}} \delta_{j k_{1}}\right) \\
& =\delta_{1} C\left(\phi_{k_{1} j_{1}}+\phi_{j_{1}} \delta_{j k_{1}}-\phi_{j k_{1} j_{1}}-\phi_{j_{1}} \delta_{j k_{1}}\right) \\
& =0 .
\end{aligned}
$$

For any term $A$ (of the mentioned type) in the above sum, there exists another term $B$, uniquely determined, which cancels out with $A$.

Concerning the terms of the second type, consider the summands

$$
E=\delta_{3}\left(\psi_{r s j}+\psi_{j} \delta_{r s}\right) D \quad \text { and } \quad F=\delta_{4}\left(\psi_{j s r}+\psi_{r} \delta_{j s}\right) D,
$$

where

and

$$
\delta_{3}=\delta\left(\begin{array}{c}
r, j, j_{1}, j_{2}, \ldots, j_{i-2} \\
s, k, k_{1}, k_{2}, \ldots, k_{i-2}
\end{array}\right), \quad \delta_{4}=\delta\left(\begin{array}{c}
j, r, j_{1}, j_{2}, \ldots, j_{i-2} \\
s, k, k_{1}, k_{2}, \ldots, k_{i-2}
\end{array}\right)
$$

$$
D=\left(\phi_{j_{1} k_{1}}+\phi \delta_{j_{1} k_{1}}\right) \cdots\left(\phi_{j_{i-2} k_{i-2}}+\phi \delta_{j_{i-2} k_{i-2}}\right) .
$$

Again, it is clear that $\delta_{3}=-\delta_{4}$, and by the same reasoning as before we get $E+F=0$, which concludes the proof.

Remark 2.4. As a consequence of Lemma 2.2, together with the divergence theorem applied twice on the sphere and the definition (10) of the matrix $Q$, it is easy to prove that, for $h \in \mathfrak{C}$ and $f, \phi \in C^{2}\left(\mathbb{S}^{n-1}\right)$,

$$
\begin{aligned}
\int_{\mathbb{S}^{n-1}} f S_{i}^{k j}(Q(h)) q_{k j}(\phi) d \mathcal{H}^{n-1} & =\int_{\mathbb{S}^{n-1}}\left(f \phi \operatorname{trace}\left(S_{i}^{k j}(Q(h))\right)+f S_{i}^{k j}(Q(h)) \phi_{k j}\right) d \mathcal{H}^{n-1} \\
& =\int_{\mathbb{S}^{n-1}}\left(f \phi \operatorname{trace}\left(S_{i}^{k j}(Q(h))\right)-S_{i}^{k j}(Q(h)) f_{j} \phi_{k}\right) d \mathcal{H}^{n-1} \\
& =\int_{\mathbb{S}^{n-1}}\left(f \phi \operatorname{trace}\left(S_{i}^{k j}(Q(h))\right)+\phi S_{i}^{k j}(Q(h)) f_{k j}\right) d \mathcal{H}^{n-1} \\
& =\int_{\mathbb{S}^{n-1}} \phi S_{i}^{k j}(Q(h)) q_{k j}(f) d \mathcal{H}^{n-1} .
\end{aligned}
$$

By Lemma 2.3, the same conclusion holds if we replace the matrix $\left(S_{i}^{j k}(Q(h))\right)_{j, k=1, \ldots, n-1}$ by the matrix $\left(S_{i}^{j k, r s}(Q(h)) q_{r s}(\phi)\right)_{j, k=1 \ldots, n-1}$. Note that here we assume that $\phi \in C^{2}\left(\mathbb{S}^{n-1}\right)$, while Lemma 2.2 and Lemma 2.3 are stated for functions of class $C^{3}$. The extension follows by a straightforward approximation argument.

2.4. Mollification. We recall a standard method to approximate continuous functions on the unit sphere by smooth functions. Let $\psi: \mathbb{R} \rightarrow[0, \infty)$ be a function of class $C^{\infty}$ with $\operatorname{sprt}(\psi) \subset$ $[-1,1]$ and $\psi(0)>0$. Then, for $k \in \mathbb{N}$, we define $\omega_{k}: \mathbf{O}(n) \rightarrow[0, \infty)$ by $\omega_{k}(\rho):=c_{k}$. $\psi\left(k^{2} \cdot\|\rho-\mathrm{id}\|^{2}\right)$, where $\mathbf{O}(n)$ is the group of rotations of $\mathbb{R}^{n}$ endowed with the Haar probability measure $\nu$, "id" is the identity element in $\mathbf{O}(n)$ and $c_{k}$ is chosen such that $\int_{\mathbf{O}(n)} \omega_{k}(\rho) \nu(d \rho)=1$. As a composition of $C^{\infty}$ maps, $\omega_{k}$ is of class $C^{\infty}$. The following lemma is standard. 
Lemma 2.5. Let $f \in C\left(\mathbb{S}^{n-1}\right)$. Then, for $k \in \mathbb{N}$, the function $f_{k}: \mathbb{S}^{n-1} \rightarrow \mathbb{R}$ defined by

$$
f_{k}(u):=\int_{\mathbf{O}(n)} f(\rho u) \omega_{k}(\rho) \nu(d \rho), \quad u \in \mathbb{S}^{n-1},
$$

is of class $C^{\infty}\left(\mathbb{S}^{n-1}\right)$, and the sequence $\left(f_{k}\right)_{k \in \mathbb{N}}$ converges to $f$ uniformly on $\mathbb{S}^{n-1}$.

\section{CONDITIONS FOR MONOTONICITY}

In this section we prove Theorems 1.2 and 1.4 We recall that $F$ is said to be "monotonic", when $F$ is increasing with respect to set inclusion (see (3)).

Let $K \in \mathcal{K}^{n}$ be of class $C_{+}^{2}$ and let $h$ be its support function, hence $h \in \mathfrak{C}$. If $\phi \in C^{2}\left(\mathbb{S}^{n-1}\right)$, then there exists $\epsilon>0$ such that

$$
h_{s}:=h+s \phi \in \mathfrak{C} \quad \text { for every } s \text { such that }|s| \leq \epsilon .
$$

Hence, for every $s \in[-\epsilon, \epsilon]$ there exists a convex body $K_{s}$ of class $C_{+}^{2}$ such that $h_{s}=h_{K_{s}}$ (by Proposition 2.1). Note that (9) implies that $\phi \geq 0$ if and only if $K_{s_{1}} \subset K_{s_{2}}$ whenever $-\epsilon \leq s_{1} \leq s_{2} \leq \epsilon$.

The quantity $\mathbf{F}\left(K_{s}\right)$ is well defined for $|s| \leq \epsilon$, and its derivative at $s=0$ is given by

$$
\left.\frac{d}{d s} \mathbf{F}\left(K_{s}\right)\right|_{s=0}=\int_{\mathbb{S}^{n-1}} f S_{i}^{k j}(Q(h)) q_{k j}(\phi) d \mathcal{H}^{n-1} .
$$

Next assume that $\mathbf{F}$ is monotonic and let $\phi$ be non-negative on $\mathbb{S}^{n-1}$. Then $s \mapsto \mathbf{F}\left(K_{s}\right)$ is an increasing function for $|s| \leq \epsilon$ so that

$$
\int_{\mathbb{S}^{n-1}} f S_{i}^{k j}(Q(h)) q_{k j}(\phi) d \mathcal{H}^{n-1} \geq 0 .
$$

Conversely, assume that (16) holds for every $h \in \mathfrak{C}$ and every non-negative $\phi \in C^{2}\left(\mathbb{S}^{n-1}\right)$. Let $K$ and $L$ be convex bodies of class $C_{+}^{2}$ such that $K \subset L$ and define

$$
H(s)=\mathbf{F}((1-s) K+s L), \quad s \in[0,1] .
$$

As above we get

$$
H^{\prime}(s)=\int_{\mathbb{S}^{n-1}} f S_{i}^{k j}\left(Q\left(h_{s}\right)\right) q_{k j}\left(h_{L}-h_{K}\right) d \mathcal{H}^{n-1},
$$

where $h_{s}=(1-s) h_{K}+s h_{L}$. Since $K \subset L$, we have $h_{L}-h_{K} \geq 0$ on $\mathbb{S}^{n-1}$. If we apply (16) with $\phi=h_{L}-h_{K}$, we obtain that $H$ is increasing. Hence $\mathbf{F}(K)=H(0) \leq H(1)=\mathbf{F}(L)$. This means that $\mathbf{F}$ is monotonic if restricted to convex bodies of class $C_{+}^{2}$; but as convex bodies of class $C_{+}^{2}$ are dense in $\mathcal{K}^{n}$ and $\mathbf{F}$ is continuous, we deduce that $\mathbf{F}$ is monotonic on $\mathcal{K}^{n}$. Thus we have proved the following statement.

Proposition 3.1. Assume that $i \in\{1, \ldots, n-1\}$ and $\mathbf{F}$ is given by (2) with $f \in C\left(\mathbb{S}^{n-1}\right)$. Then $\mathbf{F}$ is monotonic on $\mathcal{K}^{n}$ if and only if

$$
\int_{\mathbb{S}^{n-1}} f S_{i}^{k j}(Q(h)) q_{k j}(\phi) d \mathcal{H}^{n-1} \geq 0
$$

for all $h \in \mathfrak{C}$ and all $\phi \in C^{2}\left(\mathbb{S}^{n-1}\right)$ with $\phi \geq 0$ on $\mathbb{S}^{n-1}$. 
Assume that $f \in C^{2}\left(\mathbb{S}^{n-1}\right)$ and that $\mathbf{F}$ is monotonic. Then Remark 2.4 implies that in (17) the roles of $f$ and $\phi$ can be interchanged so that

$$
\int_{\mathbb{S}^{n-1}} \phi S_{i}^{k j}(Q(h)) q_{k j}(f) d \mathcal{H}^{n-1} \geq 0
$$

for all $h \in \mathfrak{C}$ and all $\phi \in C^{2}\left(\mathbb{S}^{n-1}\right)$ with $\phi \geq 0$ on $\mathbb{S}^{n-1}$. From this we infer the pointwise condition

$$
S_{i}^{k j}(Q(h, u)) q_{k j}(f, u) \geq 0
$$

for all $h \in \mathfrak{C}$ and $u \in \mathbb{S}^{n-1}$.

The converse is obviously true as well, that is, (18) implies the integral condition (17).

Proposition 3.2. Assume that $i \in\{1, \ldots, n-1\}$ and $\mathbf{F}$ is given by (2) with $f \in C^{2}\left(\mathbb{S}^{n-1}\right)$. Then $\mathbf{F}$ is monotonic on $\mathcal{K}^{n}$ if and only if (18) holds.

In order to further investigate condition (18), we need the following result.

Lemma 3.3. Let $A \in \operatorname{Sym}(n-1), A>0$, and let $u \in \mathbb{S}^{n-1}$. Then there exists a (symmetric) convex body $K \in \mathcal{K}^{n}$ of class $C_{+}^{2}$ such that

$$
Q\left(h_{K}, u\right)=A \text {. }
$$

Proof. We first consider the case $u=(0, \ldots, 0,1)$ and $A=\operatorname{diag}\left\{A_{1}, \ldots, A_{n-1}\right\}, A_{k}>0$ for every $k=1, \ldots, n-1$. We set $A_{n}=1$. The function $\bar{h}: \mathbb{R}^{n} \rightarrow \mathbb{R}$ defined by

$$
\bar{h}(x)=h\left(x_{1}, \ldots, x_{n}\right)=\left(\sum_{k=1}^{n} A_{k} x_{k}^{2}\right)^{1 / 2}
$$

is convex, and it is the 1-homogeneous extension of the support function $h=h_{\mathcal{E}}$ of an ellipsoid $\mathcal{E}$. For $x \neq 0$ we have

$$
\frac{\partial \bar{h}}{\partial x_{i}}(x)=\frac{A_{i} x_{i}}{\bar{h}(x)}
$$

and

$$
\frac{\partial^{2} \bar{h}}{\partial x_{i} \partial x_{j}}(x)=\frac{A_{i} \delta_{i j}}{\bar{h}(x)}-\frac{A_{i} A_{j} x_{i} x_{j}}{\bar{h}^{3}(x)}
$$

The (Euclidean) Hessian matrix of $\bar{h}$ at $u$ is

$$
D^{2} \bar{h}(u)=\left(\begin{array}{ccccc}
A_{1} & 0 & \ldots & 0 & 0 \\
0 & A_{2} & \ldots & 0 & 0 \\
\vdots & \vdots & \ddots & 0 & 0 \\
0 & \ldots & 0 & A_{n-1} & 0 \\
0 & 0 & \cdots & 0 & 0
\end{array}\right) .
$$

To compute the covariant derivatives of $h$, we can use the usual partial derivatives of $\bar{h}$ (see [11 §2.5] and also [3, Appendix A.2]) to obtain that

$$
Q(h, u)=\operatorname{diag}\left\{A_{1}, \ldots, A_{n-1}\right\},
$$


which finishes the proof in the case where $A>0$ is diagonal and $u=(0, \ldots, 0,1)$. In the general case, let $T$ be an orthogonal $(n-1) \times(n-1)$ matrix such that $T A T^{t}=\operatorname{diag}\left\{A_{1}, \ldots, A_{n-1}\right\}$. Choose a coordinate system such that $u=T((0, \ldots, 0,1))$, and repeat the above construction of the ellipsoid $\mathcal{E}$ for the matrix $T A T^{t}$ with respect to such a system. Then we have that $T A T^{t}=Q(h,(0, \ldots, 0,1))$. Using again the Euclidean derivatives to calculate the covariant derivatives (see [11, §2.5] and also [3, Appendix A.2]), it is not difficult to see that $A=$ $T^{t} Q(h,(0, \ldots, 0,1)) T=Q(h, T(0, \ldots, 0,1))=Q(h, u)$, which concludes the proof.

By Proposition 3.1 and the above lemma, we immediately obtain the following result.

Proposition 3.4. Assume that $i \in\{1, \ldots, n-1\}$ and $\mathbf{F}$ is given by (2) with $f \in C^{2}\left(\mathbb{S}^{n-1}\right)$. Then $\mathbf{F}$ is monotonic in $\mathcal{K}^{n}$ if and only if

$$
S_{i}^{k j}(A) q_{k j}(f, u)=\operatorname{tr}\left(\left(S_{i}^{k j}(A)\right) \cdot Q(f, u)\right) \geq 0
$$

for all $A \in \operatorname{Sym}(n-1), A>0$, and $u \in \mathbb{S}^{n-1}$.

Next we further study condition (19). Let $N=n-1$. Given the matrix $B=\operatorname{diag}\left\{b_{1}, \ldots, b_{N}\right\}$, we write $\operatorname{diag}\left\{\hat{b_{j}}\right\}$ to denote the $(N-1) \times(N-1)$ matrix $\operatorname{diag}\left\{b_{1}, \ldots b_{j-1}, b_{j+1}, \ldots, b_{N}\right\}$ obtained from $B$ by removing $b_{j}$ from the diagonal. We notice, that if $A \in \operatorname{Sym}(N)$ has the eigenvalues $\lambda_{1}, \ldots, \lambda_{N}$, then the matrix $\left(S_{i}^{k j}(A)\right)$ has the eigenvalues $\frac{\partial S_{i}(A)}{\partial \lambda_{\ell}}=S_{i-1}\left(\operatorname{diag}\left\{\hat{\lambda_{\ell}}\right\}\right)$, $\ell=1, \ldots, N$ (see [10, Proposition 1.4.1]). For a fixed $u \in \mathbb{S}^{n-1}$, we denote by $M$ the matrix $Q(f, u) \in \operatorname{Sym}(N)$. By a proper choice of the coordinate system, we may assume that $M$ is diagonal, $M=\operatorname{diag}\left\{\mu_{1}, \ldots, \mu_{N}\right\}$, and that $\left(S_{i}^{k j}(A)\right)$ is diagonal as well. Therefore we can restate condition (19) in the form

$$
\sum_{j=1}^{N} \mu_{j} S_{i-1}\left(\operatorname{diag}\left\{\hat{\lambda_{j}}\right\}\right)=\operatorname{tr}\left(\left(S_{i}^{k j}(A)\right) M\right) \geq 0
$$

for every $A=\operatorname{diag}\left\{\lambda_{1}, \ldots, \lambda_{N}\right\}>0$.

By a standard continuity argument the latter is equivalent to

$$
\sum_{j=1}^{N} \mu_{j} S_{i-1}\left(\operatorname{diag}\left\{\hat{\lambda_{j}}\right\}\right)=\operatorname{tr}\left(\left(S_{i}^{k j}(A)\right) M\right) \geq 0
$$

for every $A=\operatorname{diag}\left\{\lambda_{1}, \ldots, \lambda_{N}\right\} \geq 0$.

Using its equivalent form (20), we will prove that (19) for the matrix $M$ is equivalent to condition $\left(\mathbf{M}_{i}\right)$ expressed by (5) in the introduction.

Lemma 3.5. Let $i \in\{1, \ldots, N\}$ and let $M \in \operatorname{Sym}(N)$. Then condition (20) holds if and only if

$$
\sum_{j \in J} \mu_{j} \geq 0
$$

for all $J \subset\{1, \ldots, N\}$ of cardinality $|J|=N-i+1$.

Proof. It is straightforward to prove that condition (20) implies (21) by evaluating the inequality for positive semidefinite matrices $A=\operatorname{diag}\left\{\lambda_{1}, \ldots, \lambda_{N}\right\}$ with $\lambda_{k} \in\{0,1\}$ for $k=1, \ldots, N$. 
Indeed, it is enough to consider all such matrices where $N-i+1$ elements in the diagonal vanish and the remaining $i-1$ entries are equal to one.

For the converse, observe that

$$
\begin{aligned}
\sum_{j=1}^{N} \mu_{j} S_{i-1}\left(\operatorname{diag}\left\{\hat{\lambda_{j}}\right\}\right) & =\sum_{j=1}^{N} \mu_{j} \sum_{|I|=i-1}\left[\left(\prod_{i \in I} \lambda_{i}\right) \mathbf{1}_{\{j \notin I\}}\right] \\
& =\sum_{|I|=i-1} \sum_{j=1}^{N} \mu_{j} \mathbf{1}_{\{j \notin I\}}\left(\prod_{i \in I} \lambda_{i}\right) \\
& =\sum_{|I|=i-1}\left(\prod_{i \in I} \lambda_{i}\right) \sum_{j=1}^{N} \mu_{j} \mathbf{1}_{\{j \notin I\}} \\
& =\sum_{|I|=i-1}\left(\prod_{i \in I} \lambda_{i}\right) \sum_{j \notin I} \mu_{j} .
\end{aligned}
$$

Since $\lambda_{k} \geq 0$ for all $1 \leq k \leq N$, using (21) we obtain (20).

The above lemma and Proposition 3.4 provide the proof of Theorem 1.2

Next we proceed to prove Theorem 1.4 with the help of the regularization procedure presented in the previous section.

Proof of Theorem 1.4 We assume that the functional $\mathbf{F}$ is defined as in (2) with $f \in C\left(\mathbb{S}^{n-1}\right)$ and that it is monotonic. Then, for every $k \in \mathbb{N}$, let $f_{k}$ be defined by (14) as in Lemma 2.5 and let $\mathbf{F}_{k}$ be the functional given by (2) with $f$ replaced by $f_{k}$. Then $\mathbf{F}_{k}$ is monotonic as well. Indeed, let $K$ and $L$ be convex bodies of class $C_{+}^{2}$ with support functions $h_{K}$ and $h_{L}$, respectively, and assume that $K \subset L$. Then

$$
\begin{aligned}
& \mathbf{F}_{k}(K)-\mathbf{F}_{k}(L) \\
& =\int_{\mathbb{S}^{n-1}} f_{k}(u)\left(S_{i}\left(Q\left(h_{K}, u\right)\right)-S_{i}\left(Q\left(h_{L}, u\right)\right) \mathcal{H}^{n-1}(d u)\right. \\
& =\int_{\mathbf{O}(n)} \omega_{k}(\rho) \int_{\mathbb{S}^{n-1}} f(\rho u)\left(S_{i}\left(Q\left(h_{K}, u\right)\right)-S_{i}\left(Q\left(h_{L}, u\right)\right) \mathcal{H}^{n-1}(d u) \nu(d \rho) .\right.
\end{aligned}
$$

Now, for each $\rho \in \mathbf{O}(n)$, we have

$$
\begin{aligned}
& \int_{\mathbb{S}^{n-1}} f(\rho u)\left(S_{i}\left(Q\left(h_{K}, u\right)\right)-S_{i}\left(Q\left(h_{L}, u\right)\right) \mathcal{H}^{n-1}(d u)\right. \\
& =\int_{\mathbb{S}^{n-1}} f(u)\left(S_{i}\left(Q\left(h_{K}, \rho^{-1} u\right)\right)-S_{i}\left(Q\left(h_{L}, \rho^{-1} u\right)\right) \mathcal{H}^{n-1}(d u)\right. \\
& =\int_{\mathbb{S}^{n-1}} f(u)\left(S_{i}\left(Q\left(h_{\rho K}, u\right)\right)-S_{i}\left(Q\left(h_{\rho L}, u\right)\right) \mathcal{H}^{n-1}(d u)\right. \\
& =F(\rho K)-F(\rho L) \leq 0,
\end{aligned}
$$

where in the last inequality we have used $\rho K \subset \rho L$ and the monotonicity of $\mathbf{F}$. 
This proves that $\mathbf{F}_{\mathbf{k}}$ is monotone for every $k \in \mathbb{N}$. Since $f_{k}$ is of class $C^{2}\left(\mathbb{S}^{n-1}\right)$, it satisfies condition $(\mathbf{M})_{i}$ by Theorem 1.2, and this concludes the proof of Theorem 1.4

Remark 3.6. In the introduction, we already pointed out the meaning of condition $(\mathbf{M})_{i}$ in the special cases $i=n-1$ and $i=1$. Let us consider the case $i=2$. It can be proved that for every $A$ and $B$ in $\operatorname{Sym}(n-1)$ we have

$$
\operatorname{tr}\left(S_{2}^{k j}(A) \cdot B\right)=\operatorname{tr}\left(S_{2}^{k j}(B) \cdot A\right) .
$$

Hence, if $f \in C^{2}\left(\mathbb{S}^{n-1}\right)$, condition (19) becomes

$$
\operatorname{tr}\left(A\left(S_{2}^{k j}(Q(f, u))\right)\right) \geq 0 \quad \text { for every } A \in \operatorname{Sym}(n-1), A>0,
$$

for every $u \in \mathbb{S}^{n-1}$. This is equivalent to the condition $\left(S_{2}^{k j}(Q(f, u))\right) \geq 0$ for every $u \in \mathbb{S}^{n-1}$.

\section{CONDITIONS FOR CONCAVITY}

This section is devoted to the proof of Theorem 1.5 and some of its extensions. We consider a functional $\mathbf{F}$ of the form (2), and we assume that $\mathbf{F}$ is non-negative on $\mathcal{K}^{n}$ and satisfies the Brunn-Minkowski inequality

$$
\mathbf{F}((1-t) K+t L)^{1 / i} \geq(1-t) \mathbf{F}(K)^{1 / i}+t \mathbf{F}(L)^{1 / i}
$$

for all $K, L \in \mathcal{K}^{n}$ and $t \in[0,1]$. As noted in the introduction, if $i=1$ then $\mathbf{F}$ is linear with respect to Minkowski addition and (22) is satisfied (with equality) for every $f$. Moreover, the case $i=n-1$ has been settled in [3]. Hence we will consider the cases where $2 \leq i \leq n-2$ in the following.

Proof of Theorem 1.5. As a first step towards the proof, we show that if $\mathbf{F}$ is not identically zero, then $\mathbf{F}(K)>0$ for every $K \in C_{+}^{2}$. Indeed, as $C_{+}^{2}$ bodies are dense in $\mathcal{K}^{n}$ and $\mathbf{F}$ is continuous, there exists at least one of them, denoted by $K_{0}$, such that $\mathbf{F}\left(K_{0}\right)>0$. On the other hand, for any other $K \in C_{+}^{2}$, a suitable rescaled version of $K_{0}$ is a summand of $K$, i.e., there exists $K^{\prime} \in \mathcal{K}^{n}$ and $\lambda \in(0,1)$ such that $K=(1-\lambda) K^{\prime}+\lambda K_{0}$ (see [11, Corollary 3.2.13]). From (22) it follows immediately that $\mathbf{F}(K) \geq \lambda^{i} \mathbf{F}\left(K_{0}\right)>0$. On the other hand, if $\mathbf{F}$ is identically zero, then, in particular, it is monotonic so that condition $(\mathbf{M})_{i}$ holds (cf. Theorem 1.2). From now on we will assume that $\mathbf{F}$ is strictly positive for $C_{+}^{2}$ convex bodies.

Consider $K \in \mathcal{K}^{n}$ of class $C_{+}^{2}$ and denote by $h$ its support function, then $h \in \mathfrak{C}$. For $\phi \in$ $C^{\infty}\left(\mathbb{S}^{n-1}\right)$, let $\epsilon>0$ be such that

$$
h_{s}:=h+s \phi \in \mathfrak{C} \quad \text { for every } s \text { such that }|s| \leq \epsilon .
$$

For $s \in[-\epsilon, \epsilon]$ let $K_{s} \in \mathfrak{C}$ be such that $h_{s}=h_{K_{s}}$. We compute the first and second derivatives of $H(s):=\mathbf{F}\left(K_{s}\right)$ at $s=0$. In fact, we already saw in (15) that

$$
H^{\prime}(s)=\int_{\mathbb{S}^{n-1}} f S_{i}^{k j}\left(Q\left(h_{s}\right)\right) q_{k j}(\phi) d \mathcal{H}^{n-1}
$$


(recall that we use the convention that we sum over repeated indices). As $f \in C^{2}\left(\mathbb{S}^{n-1}\right)$, applying Lemma 2.2 to the last equality we get

$$
H^{\prime}(s)=\int_{\mathbb{S}^{n-1}} \phi S_{i}^{k j}\left(Q\left(h_{s}\right)\right) q_{k j}(f) d \mathcal{H}^{n-1} .
$$

Differentiating once more with respect to $s$ (at $s=0$ ) and using the notation introduced in Section 2.2, we obtain

$$
H^{\prime \prime}(0)=\int_{\mathbb{S}^{n-1}} \phi S_{i}^{k j, r s}(Q(h)) q_{k j}(f) q_{r s}(\phi) d \mathcal{H}^{n-1} .
$$

Since $K_{(1-\lambda) s+\lambda s^{\prime}}=(1-\lambda) K_{s}+\lambda K_{s^{\prime}}$, inequality (22) yields that the function $s \mapsto G(s):=$ $H(s)^{1 / i}$ is concave for $s \in[-\epsilon, \epsilon]$. Since $\mathbf{F}(K)>0, H(0)>0$ and $G$ is twice differentiable at $s=0$, we conclude that $G^{\prime \prime}(0) \leq 0$, and hence

$$
H(0) H^{\prime \prime}(0)-\frac{i-1}{i} H^{\prime}(0)^{2} \leq 0 .
$$

This implies

$$
\begin{aligned}
\mathbf{F}(K) \cdot \int_{\mathbb{S}^{n-1}} \phi S_{i}^{k j, r s}(Q(h)) q_{k j}(f) q_{r s}(\phi) d \mathcal{H}^{n-1} \\
\quad \leq \frac{i-1}{i}\left(\int_{\mathbb{S}^{n-1}} \phi S_{i}^{k j}(Q(h)) q_{k j}(f) d \mathcal{H}^{n-1}\right)^{2}
\end{aligned}
$$

for every $h \in \mathfrak{C}$ and $\phi \in C^{2}\left(\mathbb{S}^{n-1}\right)$. For brevity, we set

$$
M=\left(m_{r s}(u)\right)_{r, s=1, \ldots, n-1}:=\mathbf{F}(K) \cdot\left(S_{i}^{k j, r s}(Q(h, u)) q_{k j}(f, u)\right)_{r, s=1, \ldots, n-1}
$$

for $u \in \mathbb{S}^{n-1}$. Integrating by parts and using Lemma 2.3, we rewrite (23) in the form

$$
\int_{\mathbb{S}^{n-1}} \phi^{2} \operatorname{trace}(M) d \mathcal{H}^{n-1} \leq \int_{\mathbb{S}^{n-1}} m_{r s} \phi_{r} \phi_{s} d \mathcal{H}^{n-1}+\left(\int_{\mathbb{S}^{n-1}} \phi g d \mathcal{H}^{n-1}\right)^{2},
$$

for every $\phi \in C^{\infty}\left(\mathbb{S}^{n-1}\right)$, where

$$
g(u)=\sqrt{\frac{i-1}{i}} S_{i}^{k j}(Q(h, u)) q_{k j}(f, u), \quad u \in \mathbb{S}^{n-1} .
$$

The next step, which is the crucial part of the proof, is to show that (25) implies the pointwise matrix condition

$$
\left(S_{i}^{k j, r s}(Q(h, u)) q_{k j}(f, u)\right)_{r, s=1, \ldots, n-1} \geq 0
$$

for all $u \in \mathbb{S}^{n-1}$. For this, we need a result similar to Lemma 3.3 in [3], which is Lemma 4.1] presented at the end of this proof. This result applied to (25) immediately gives (27). In particular, as $Q(h) \geq 0$ on $\mathbb{S}^{n-1}$, we get

$$
S_{i}^{k j, r s}(Q(h, u)) q_{k j}(f, u) q_{r s}(h, u) \geq 0
$$

for all $u \in \mathbb{S}^{n-1}$. On the other hand, by the homogeneity of the elementary symmetric function $S_{i}$ and its derivatives, we have

$$
S_{i}^{k j, r s}(Q(h)) q_{r s}(h)=c \cdot S_{i}^{k j}(Q(h))
$$


for some constant $c>0$ and for every $k, j \in\{1, \ldots, n-1\}$. Hence

$$
S_{i}^{k j}(Q(h, u)) q_{k j}(f, u) \geq 0, \quad u \in \mathbb{S}^{n-1},
$$

for every $h \in \mathfrak{C}$, that is, condition (18), which is equivalent to the monotonicity of $\mathbf{F}$ and also to condition $(\mathbf{M})_{i}$ (see Proposition 3.1 and comments below). Hence Theorem 1.5] is proved.

Lemma 4.1. For $r, s \in\{1, \ldots, n-1\}$ and $m_{r s} \in C\left(\mathbb{S}^{n-1}\right)$ let $M:=\left(m_{r s}\right)_{r, s=1, \ldots, n-1}$ and $g \in C\left(\mathbb{S}^{n-1}\right)$. If inequality (25) holds for every $\phi \in C^{\infty}\left(\mathbb{S}^{n-1}\right)$, then $M(u) \geq 0$ for every $u \in \mathbb{S}^{n-1}$.

The proof follows the lines of that of Lemma 3.3 in [3]; we provide it for the reader's convenience.

Proof. By standard approximation (25) can be extended to every $\phi \in C\left(\mathbb{S}^{n-1}\right)$ which is Lipschitz on $\mathbb{S}^{n-1}$ (interpreting the first derivatives of $\phi$ as functions defined $\mathcal{H}^{n-1}$-a.e. on $\mathbb{S}^{n-1}$ ).

We proceed by contradiction. Let us assume there exist $\bar{u} \in \mathbb{S}^{n-1}$ and $\bar{v}=\left(\bar{v}_{1}, \ldots, \bar{v}_{n-1}\right) \in$ $\mathbb{R}^{n-1}$ such that

$$
\sum_{r, s=1}^{n-1} m_{r s}(\bar{u}) \bar{v}_{r} \bar{v}_{s}<0 .
$$

Without loss of generality we may assume $\bar{u}=(0, \ldots, 1)$ and $\bar{v}=(1, \ldots, 0)$. Then we have

$$
\sum_{r, s=1}^{n-1} m_{r s}(\bar{u}) \bar{v}_{r} \bar{v}_{s}=m_{11}(\bar{u})<0 .
$$

We identify $H:=\left\{x=\left(x_{1}, \ldots, x_{n}\right) \in \mathbb{R}^{n}: x_{n}=0\right\}$ with $\mathbb{R}^{n-1}$ and, for $\rho \in(0,1)$, we set

$$
\begin{aligned}
& D_{\rho}:=\left\{\left(x_{1}, \ldots, x_{n-1}\right) \in \mathbb{R}^{n-1}:\left|x_{i}\right| \leq \rho, i=1, \ldots, n-1\right\}, \\
& \tilde{D}_{\rho}:=\left\{u=\left(u_{1}, \ldots, u_{n}\right) \in \mathbb{S}^{n-1}: u_{n}>0,\left(u_{1}, \ldots, u_{n-1}\right) \in D_{\rho}\right\} .
\end{aligned}
$$

We construct a Lipschitz function $\phi$ such that inequality (25) fails to be true. Define first $\bar{g}$ : $[-1,1] \rightarrow \mathbb{R}_{+}$as $\bar{g}(t)=1-|t|$, and denote by $g(t)$ the periodic extension of $\bar{g}$ to the whole real line. Let $\epsilon>0$ and define $g_{\epsilon}(x)=\epsilon g(x / \epsilon)$. Notice that $g_{\epsilon} \searrow 0$ uniformly on $\mathbb{R}$, as $\epsilon$ tends to 0 . Let

$$
G(t):= \begin{cases}1, & \text { for } t \in[-1 / 2,1 / 2], \\ 0, & \text { for }|t| \geq 1, \\ \text { linear extension, } & \text { otherwise. }\end{cases}
$$

Then $G$ is a bounded Lipschitz function in $\mathbb{R}$. Let us fix $\rho \in(0,1)$. The function

$$
\Phi_{\epsilon}\left(x_{1}, \ldots, x_{n-1}\right)=g_{\epsilon}\left(x_{1}\right) G\left(x_{1} / \rho\right) \ldots G\left(x_{n-1} / \rho\right), \quad\left(x_{1}, \ldots, x_{n}\right) \in D_{\rho},
$$

is a bounded Lipschitz function in $D_{\rho}$ and $\operatorname{sprt}\left(\Phi_{\epsilon}\right) \subset D_{\rho}$. For $k \neq 1$ we have

$$
\frac{\partial \Phi_{\epsilon}}{\partial x_{k}}\left(x_{1}, \ldots, x_{n-1}\right)=\frac{1}{\rho} g_{\epsilon}\left(x_{1}\right) G^{\prime}\left(x_{k} / \rho\right) \prod_{j \neq k} G\left(x_{j} / \rho\right),
$$


for $\mathcal{H}^{n-1}$-a.e. $\left(x_{1}, \ldots, x_{n-1}\right) \in D_{\rho}$. As $G \leq 1,\left|G^{\prime}\right| \leq 2$ and $\left|g_{\epsilon}\right| \leq \epsilon$ in $\mathbb{R}$,

$$
\left|\frac{\partial \Phi_{\epsilon}}{\partial x_{k}}\right| \leq \frac{2 \epsilon}{\rho} \quad \mathcal{H}^{n-1} \text {-a.e. in } D_{\rho}
$$

and then

$$
\lim _{\epsilon \searrow 0} \frac{\partial \Phi_{\epsilon}}{\partial x_{k}}=0, \quad \mathcal{H}^{n-1} \text {-a.e. in } D_{\rho} .
$$

On the other hand, for $k=1$ and for $\mathcal{H}^{n-1}$-a.e. $\left(x_{1}, \ldots, x_{n-1}\right) \in D_{\rho}$

$$
\frac{\partial \Phi_{\epsilon}}{\partial x_{1}}\left(x_{1}, \ldots, x_{n-1}\right)=\frac{1}{\rho} g_{\epsilon}\left(x_{1}\right) G^{\prime}\left(x_{1} / \rho\right) \prod_{j>1} G\left(x_{j} / \rho\right)+g_{\epsilon}^{\prime}\left(x_{1}\right) \prod_{j=1}^{n-1} G\left(x_{j} / \rho\right) .
$$

As $\left|g_{\epsilon}^{\prime}\right|=1$ holds $\mathcal{H}^{1}$-a.e. in $\mathbb{R}$, we get

$$
\left|\frac{\partial \Phi_{\epsilon}}{\partial x_{1}}\right|\left(x_{1}, \ldots, x_{n-1}\right) \longrightarrow \prod_{j=1}^{n-1} G\left(x_{j} / \rho\right) \text { for } \mathcal{H}^{n-1} \text {-a.e. }\left(x_{1}, \ldots, x_{n-1}\right) \in D_{\rho},
$$

as $\epsilon \searrow 0$. In particular, the above limit equals one $\mathcal{H}^{n-1}$-a.e. in $D_{\rho / 2}$. Consider the function

$$
\phi_{\epsilon}(u)=\phi_{\epsilon}\left(u_{1}, \ldots, u_{n}\right):=\Phi_{\epsilon}\left(u_{1}, \ldots, u_{n-1}\right), \quad u \in \tilde{D}_{\rho},
$$

and extend $\phi_{\epsilon}$ to be zero in the rest of the unit sphere $\mathbb{S}^{n-1}$. In the sequel, for $u=\left(u_{1}, \ldots, u_{n}\right) \in$ $\tilde{D}_{\rho}$, we set $u^{\prime}=\left(u_{1}, \ldots, u_{n-1}\right) \in D_{\rho}$. As $\rho<1$, the support of $\phi_{\epsilon}$ is contained in the open hemisphere $\mathbb{S}^{n-1} \cap\left\{x_{n}>0\right\}$. We may take $\rho$ small enough such that there exists a local orthonormal frame of coordinates on $\tilde{D}_{\rho}$. Taking covariant derivatives with respect to this frame, by (25) we have

$$
\int_{\mathbb{S}^{n-1}} \phi_{\epsilon}^{2} \operatorname{trace}(M) d \mathcal{H}^{n-1}(u) \leq \int_{\mathbb{S}^{n-1}} \sum_{j, k=1}^{n-1}\left(\phi_{\epsilon}\right)_{j}\left(\phi_{\epsilon}\right)_{k} m_{j k} d \mathcal{H}^{n-1}+\left(\int_{\mathbb{S}^{n-1}} \phi_{\epsilon} g d \mathcal{H}^{n-1}\right)^{2} .
$$

Since $\Phi_{\epsilon}$ converges to zero uniformly as $\epsilon \searrow 0$, the same is valid for $\phi_{\epsilon}$, hence

$$
0 \leq \liminf _{\epsilon \searrow 0} \int_{\mathbb{S}^{n-1}} \sum_{j, k=1}^{n-1} m_{j k}\left(\phi_{\epsilon}\right)_{j}\left(\phi_{\epsilon}\right)_{k} d \mathcal{H}^{n-1}
$$

The covariant derivatives of $\phi_{\epsilon}$ can be computed in terms of partial derivatives of $\Phi$ with respect to Cartesian coordinates on $D_{\rho}$; in particular, there exists a $(n-1) \times(n-1)$ matrix $C=$ $\left(c_{r s}\right)_{r, s=1, \ldots, n-1}$, depending on $u$, with $c_{r s} \in C\left(D_{\rho}\right)$ for $r, s=1, \ldots, n-1$, such that

$$
\left(\phi_{\epsilon}\right)_{j}(u)=\sum_{s=1}^{n-1} c_{j s}\left(u^{\prime}\right) \frac{\partial \Phi_{\epsilon}}{\partial x_{s}}\left(u^{\prime}\right), \quad \text { for } \mathcal{H}^{n-1} \text {-a.e. } u \in \tilde{D}_{\rho} .
$$

We may assume that $C\left(\bar{u}^{\prime}\right)=C(0, \ldots, 0)$ is the identity matrix. Then, for $\mathcal{H}^{n-1}$-a.e. $u \in \tilde{D}_{\rho}$,

$$
\sum_{j, k=1}^{n-1} m_{j k}(u)\left(\phi_{\epsilon}\right)_{j}(u)\left(\phi_{\epsilon}\right)_{k}(u)=\sum_{j, k=1}^{n-1} \sum_{r, s=1}^{n-1} m_{j k}(u) c_{j s}\left(u^{\prime}\right) c_{k r}\left(u^{\prime}\right) \frac{\partial \Phi_{\epsilon}}{\partial x_{r}}\left(u^{\prime}\right) \frac{\partial \Phi_{\epsilon}}{\partial x_{s}}\left(u^{\prime}\right) \text {. }
$$


This expression is bounded, by the boundedness of the partial derivatives of $\Phi_{\epsilon}$. Moreover, by (28) and (29),

$$
\lim _{\epsilon \searrow 0} \sum_{j, k=1}^{n-1} m_{j k}(u)\left(\phi_{\epsilon}\right)_{j}(u)\left(\phi_{\epsilon}\right)_{k}(u)=\prod_{s=1}^{n-1} G^{2}\left(u_{s} / \rho\right) \sum_{j, k=1}^{n-1} m_{j k}(u) c_{j 1}\left(u^{\prime}\right) c_{k 1}\left(u^{\prime}\right) .
$$

Note that

$$
\sum_{i, j=1}^{n-1} m_{i j}(\bar{u}) c_{i 1}\left(\bar{u}^{\prime}\right) c_{j 1}\left(\bar{u}^{\prime}\right)=m_{11}(\bar{u})<0
$$

Consequently, we may choose $\rho$ sufficiently small so that

$$
\sum_{i, j=1}^{n-1} m_{i j}(u) c_{i 1}\left(u^{\prime}\right) c_{j 1}\left(u^{\prime}\right) \leq c<0, \quad u \in \tilde{D}_{\rho} .
$$

Then

$$
\begin{aligned}
& \lim _{\epsilon \searrow 0} \int_{\mathbb{S}^{n-1}} \sum_{i, j=1}^{n-1} m_{i j}\left(\phi_{\epsilon}\right)_{i}\left(\phi_{\epsilon}\right)_{j} d \mathcal{H}^{n-1} \\
& \quad=\int_{\mathbb{S}^{n-1}} \prod_{i=1}^{n-1} G^{2}\left(u_{i} / \rho\right) \sum_{i, j=1}^{n-1} m_{i j}(u) c_{i 1}\left(u^{\prime}\right) c_{j 1}\left(u^{\prime}\right) \mathcal{H}^{n-1}(d u) \\
& \quad=\int_{\tilde{D}_{\rho}} \prod_{i=1}^{n-1} G^{2}\left(u_{i} / \rho\right) \sum_{i, j=1}^{n-1} m_{i j}(u) c_{i 1}\left(u^{\prime}\right) c_{j 1}\left(u^{\prime}\right) \mathcal{H}^{n-1}(d u) \\
& \quad \leq \int_{\tilde{D}_{\rho / 2}} \sum_{i, j=1}^{n-1} m_{i j}(u) c_{i 1}\left(u^{\prime}\right) c_{j 1}\left(u^{\prime}\right) \mathcal{H}^{n-1}(d u) \\
& \leq c \mathcal{H}^{n-1}\left(\tilde{D}_{\rho / 2}\right)<0
\end{aligned}
$$

which contradicts (30).

Proof of Theorem 1.7 Proceeding as in the first part of the proof of Theorem 1.5 (but without integration by parts), we arrive at the following inequality (see (23)

$$
\begin{aligned}
\mathbf{F}(K) & \cdot \int_{\mathbb{S}^{n-1}} f S_{i}^{k j, r s}(Q(h)) q_{k j}(\phi) q_{r s}(\phi) d \mathcal{H}^{n-1} \\
\leq & \frac{i-1}{i}\left(\int_{\mathbb{S}^{n-1}} f S_{i}^{k j}(Q(h)) q_{k j}(\phi) d \mathcal{H}^{n-1}\right)^{2},
\end{aligned}
$$

for every $h \in \mathfrak{C}$ and every $\phi \in C^{\infty}\left(\mathbb{S}^{n-1}\right)$. If, in particular, $h$ is even (and hence is the support function of a centrally symmetric convex body) and $\phi$ is odd, then the right hand-side of (31) vanishes (as $f$ is even), being the integral of an odd function on $\mathbb{S}^{n-1}$. We may assume, as in the previous proof, that $\mathbf{F}(K)>0$ for every $K \in C_{+}^{2}$. Hence we get

$$
\int_{\mathbb{S}^{n-1}} f S_{i}^{k j, r s}(Q(h)) q_{k j}(\phi) q_{r s}(\phi) d \mathcal{H}^{n-1} \leq 0
$$


for every $h \in \mathfrak{C}$ even and $\phi \in C^{\infty}\left(\mathbb{S}^{n-1}\right)$ odd. Let $\psi \in C^{\infty}\left(\mathbb{S}^{n-1}\right)$ be such that its support is contained in a open hemisphere $H^{+}$, and let $\phi: \mathbb{S}^{n-1} \rightarrow \mathbb{R}$ be defined by

$$
\bar{\phi}(u)= \begin{cases}\psi(u), & \text { if } u \in H^{+} \\ -\psi(-u), & \text { if } u \in \mathbb{S}^{n-1} \backslash H^{+}\end{cases}
$$

Then $\bar{\phi} \in C^{\infty}\left(\mathbb{S}^{n-1}\right)$ is an odd function. For this choice of $\bar{\phi}$ in $(\underline{32})$, in view of the symmetry of $f$ and $h$, we get

$$
\int_{\mathbb{S}^{n-1}} f S_{i}^{k j, r s}(Q(h)) q_{k j}(\psi) q_{r s}(\psi) d \mathcal{H}^{n-1} \leq 0,
$$

for every $\psi \in C^{\infty}\left(\mathbb{S}^{n-1}\right)$ with support contained in an open hemisphere. We can now apply to $f$ the regularization procedure indicated in Section 2 in a similar way as in the proof of Theorem 1.4. As the left hand-side of (33) is linear with respect to $f$, we obtain that $f_{k}$ satisfies (33) as well, for every $k \in \mathbb{N}$. Note that the functions on which the proof of Lemma 4.1 is based are all supported in an open hemisphere. Hence we can apply this lemma to $f_{k}$ and conclude that it satisfies condition (18) for every $h \in \mathfrak{C}$ even. By Lemma 3.3 (note that the proof of this lemma requires the use of even functions only) we have that condition (19) holds and then, via Lemma 3.5, condition $(\mathbf{M})_{i}$ holds as well.

We conclude this section with the following variant of Theorem 1.5 in which the regularity assumption on $f$ is weakened, and the symmetry hypothesis appearing in 1.7 is replaced by the assumption that $f$ belongs to $W^{2,1}\left(\mathbb{S}^{n-1}\right)$, the Sobolev space of functions in $L^{1}\left(\mathbb{S}^{n-1}\right)$ having second weak derivatives in $L^{1}\left(\mathbb{S}^{n-1}\right)$.

Theorem 4.2. Let $i \in\{1, \ldots, n-1\}$, let $f \in W^{2,1}\left(\mathbb{S}^{n-1}\right)$ be continuous, and let $\mathbf{F}$ be defined as in (2). If $\mathbf{F}$ is non-negative and satisfies inequality (4), then there exists a sequence $f_{k} \in$ $C^{2}\left(\mathbb{S}^{n-1}\right), k \in \mathbb{N}$, which converges uniformly on $\mathbb{S}^{n-1}$ to $f$ such that $f_{k}$ satisfies condition $(\mathbf{M})_{i}$ for every $k \in \mathbb{N}$. In particular, $\mathbf{F}$ is monotonic.

Proof. As in the proof of Theorem 1.5, the validity of Brunn-Minkowski inequality (4) implies (25), where $M$ and $g$, defined by (24) and (26) respectively, involve weak second derivatives of $f$. Moreover, we can assume that $F$ is positive on convex bodies of class $C_{+}^{2}$. Then inequality (25) can be restated in the form

$$
\begin{gathered}
\int_{\mathbb{S}^{n-1}} \phi(u)^{2} \operatorname{trace}\left(S_{i}^{k j, r s}(Q(h, u)) q_{k j}(f, u)\right) \mathcal{H}^{n-1}(d u) \\
-\frac{i-1}{i F(K)}\left(\int_{\mathbb{S}^{n-1}} \phi(u) S_{i}^{k j}(Q(h, u)) q_{k j}(f, u) \mathcal{H}^{n-1}(d u)\right)^{2} \\
\leq \int_{\mathbb{S}^{n-1}} \sum_{i, j=1}^{n-1} S_{i}^{k j, r s}(Q(h, u)) q_{k j}(f, u) \phi_{i}(u) \phi_{j}(u) \mathcal{H}^{n-1}(d u),
\end{gathered}
$$

for all $K \in \mathcal{K}^{n}$ of class $C_{+}^{2}$ with support function $h$ and all $\phi \in C^{\infty}\left(\mathbb{S}^{n-1}\right)$. For $\rho \in \mathbf{O}(n), \rho K$ is also of class $C_{+}^{2}$ and its support function is $h \circ \rho^{-1}$. Applying now (34) with $\rho K, h \circ \rho^{-1}$, and $\phi=\phi_{\epsilon} \circ \rho^{-1}$, where $\phi_{\epsilon}$ is as in the proof of Lemma 4.1. Multiplying both sides of (34) with 
the mollifier $\omega_{l}, l \in \mathbb{N}$, integrating over the rotation group and using the rotation invariance of Hausdorff measures, we get on the right-hand side

$$
\begin{aligned}
\int_{\mathbf{O}(n)} \omega_{l}(\rho) \int_{\mathbb{S}^{n-1}} \sum_{i, j=1}^{n-1} S_{i}^{k j, r s}\left(Q\left(h \circ \rho^{-1}, \rho u\right)\right) q_{k j}(f, \rho u) \\
\times\left(\left(\phi_{\epsilon}\right) \circ \rho^{-1}\right)_{i}(\rho u)\left(\left(\phi_{\epsilon}\right) \circ \rho^{-1}\right)_{j}(\rho u) \mathcal{H}^{n-1}(d u) \nu(d \rho) \\
=\int_{\mathbf{O}(n)} \int_{\mathbb{S}^{n-1}} \sum_{i, j=1}^{n-1} S_{i}^{k j, r s}(Q(h, v)) \omega_{l}(\rho) q_{k j}\left(f_{\rho}, v\right)\left(\phi_{\epsilon}\right)_{i}(v)\left(\phi_{\epsilon}\right)_{j}(v) \mathcal{H}^{n-1}(d v) \nu(d \rho) \\
=\int_{\mathbb{S}^{n-1}} \sum_{i, j=1}^{n-1} S_{i}^{k j, r s}(Q(h, v)) q_{k j}\left(f_{l}, v\right)\left(\phi_{\epsilon}\right)_{i}(v)\left(\phi_{\epsilon}\right)_{j}(v) \mathcal{H}^{n-1}(d v) .
\end{aligned}
$$

On the other hand, we can bound the resulting two integrals on the left-hand side by

$$
\begin{aligned}
& \left.\mid \int_{\mathbf{O}(n)} \omega_{l}(\rho) \int_{\mathbb{S}^{n-1}}\left(\left(\phi_{\epsilon}\right) \circ \rho^{-1}\right)(u)\right)^{2} \operatorname{trace}\left(S_{i}^{k j, r s}\left(Q\left(h \circ \rho^{-1}, u\right)\right) q_{k j}(f, u)\right) \mathcal{H}^{n-1}(d u) \nu(d \rho) \mid \\
& \quad \leq \int_{\mathbf{O}(n)} \omega_{l}(\rho)\left\|\phi_{\epsilon}\right\|_{L^{\infty}\left(\mathbb{S}^{n-1}\right)}^{2} c_{1}(h)\|f\|_{W^{1,2}\left(\mathbb{S}^{n-1}\right)} \nu(d \rho) \\
& \quad \leq c_{2}(h, f)\left\|\phi_{\epsilon}\right\|_{L^{\infty}\left(\mathbb{S}^{n-1}\right)}^{2}
\end{aligned}
$$

and

$$
\begin{aligned}
& \left.\mid \int_{\mathbf{O}(n)} \omega_{l}(\rho) \frac{i-1}{i F(\rho K)}\left(\int_{\mathbb{S}^{n-1}}\left(\phi_{\epsilon}\right) \circ \rho^{-1}\right)(u) S_{i}^{k j}\left(Q\left(h \circ \rho^{-1}, u\right)\right) q_{k j}(f, u) \mathcal{H}^{n-1}(d u)\right)^{2} \nu(d \rho) \mid \\
& \quad \leq \int_{\mathbf{O}(n)} \omega_{l}(\rho)(\min \{F(\rho K): \rho \in \mathbf{O}(n)\})^{-1}\left\|\phi_{\epsilon}\right\|_{L^{\infty}\left(\mathbb{S}^{n-1}\right)}^{2} c_{3}(h)\|f\|_{W^{1,2}\left(\mathbb{S}^{n-1}\right)}^{2} \nu(d \rho) \\
& \quad \leq c_{4}(h, f)\left\|\phi_{\epsilon}\right\|_{L^{\infty}\left(\mathbb{S}^{n-1}\right)}^{2} .
\end{aligned}
$$

The constants $c_{1}, \ldots, c_{4}$ depend only on the parameters indicated in brackets. Here we use that the minimum $\min \{F(\rho K): \rho \in \mathbf{O}(n)\}$ is positive and depends only on $f$ and $K$, since $\rho \mapsto F(\rho K)$ is continuous and positive. From $\left\|\phi_{\epsilon}\right\|_{L^{\infty}\left(\mathbb{S}^{n-1}\right)} \rightarrow 0$ as $\epsilon \searrow 0$, we now deduce that

$$
\liminf _{\epsilon \searrow 0} \int_{\mathbb{S}^{n-1}} \sum_{i, j=1}^{n-1} S_{i}^{k j, r s}(Q(h, v)) q_{k j}\left(f_{l}, v\right)\left(\phi_{\epsilon}\right)_{i}(v)\left(\phi_{\epsilon}\right)_{j}(v) \mathcal{H}^{n-1}(d v) \geq 0
$$

for all $l \in \mathbb{N}$. Since $f_{l} \in C^{\infty}\left(\mathbb{S}^{n-1}\right)$, we can apply the argument used in the proof of Lemma4.1 to see that the matrix

$$
\left(S_{i}^{k j, r s}(Q(h, v)) q_{k j}\left(f_{l}, v\right)\right)_{r, s=1, \ldots, n-1}
$$

is positive-semidefinite for all $l \in \mathbb{N}, h \in \mathfrak{C}$ and all $v \in \mathbb{S}^{n-1}$.

From this point, we can proceed as in the proof of Theorem 1.5 after (27), obtaining that $f_{l}$ satisfies condition $(\mathbf{M})_{i}$. 


\section{Proof of TheOREM 1.9}

This section contains the proof of Theorem 1.9, and hence of Theorem 1.8, preceded by an auxiliary lemma.

For the proof we proceed by induction over the dimension $n \geq 3$. The proof uses in an essential way the special case $i=n-1$ treated in [3].

We start with an auxiliary lemma. For this, let $\delta_{x}$ denote the Dirac measure with unit mass in the point $x \in \mathbb{R}^{n}$. We denote by $V^{E}$ the mixed volume of convex bodies contained in $E$, defined on $\mathcal{K}^{\operatorname{dim} E}$.

Lemma 5.1. Let $E$ be an $(n-1)$-dimensional linear subspace in $\mathbb{R}^{n}$ with unit normal $u$. Let $K_{1}, \ldots, K_{n-2} \subset E$ be convex bodies. Let $B=B^{n} \cap E$, where $B^{n}$ is the Euclidean unit ball in $\mathbb{R}^{n}$ and $R \in \mathbb{R}, R>0$. If $\eta \subset \mathbb{S}^{n-1}$ is an arbitrary Borel set, then

$$
\begin{aligned}
& S\left(K_{1}, \ldots, K_{n-2}, B+R\left[-e_{n}, e_{n}\right] ; \eta\right) \\
& \quad=\frac{R}{n-1} S^{E}\left(K_{1}, \ldots, K_{n-2} ; \eta \cap E\right)+V^{E}\left(K_{1}, \ldots, K_{n-2}, B\right)\left(\delta_{u}+\delta_{-u}\right)(\eta) .
\end{aligned}
$$

Proof. Without loss of generality we can assume that $u=e_{n}$. Using the linearity of the surface area measures, we have that

$$
\begin{aligned}
& S\left(K_{1}, \ldots, K_{n-2}, B+R\left[0, e_{n}\right] ; \eta\right) \\
& \quad=S\left(K_{1}, \ldots, K_{n-2}, B ; \eta\right)+R S\left(K_{1}, \ldots, K_{n-2},\left[0, e_{n}\right] ; \eta\right) .
\end{aligned}
$$

Let $L \in \mathcal{K}^{n}$ be an arbitrary convex body with support function $h_{L}$ and $K_{1}, \ldots, K_{n-2} \in \mathcal{K}^{n}$. Then we have

$$
\begin{aligned}
\int_{\mathbb{S}^{n-1}} & h_{L}(u) S\left(K_{1}, \ldots, K_{n-2},\left[0, e_{n}\right] ; d u\right) \\
= & n V\left(L, K_{1}, \ldots, K_{n-2},\left[0, e_{n}\right]\right) \\
= & V^{E}\left(\left.L\right|_{E},\left.K_{1}\right|_{E}, \ldots,\left.K_{n-2}\right|_{E}\right) \\
= & \frac{1}{n-1} \int_{\mathbb{S}^{n-1}} h\left(\left.L\right|_{E}, u\right) S^{E}\left(K_{1}, \ldots, K_{n-2} ; d u\right) \\
= & \frac{1}{n-1} \int_{\mathbb{S}^{n-1}} h(L, u) \mathbf{1}_{E}(u) S^{E}\left(K_{1}, \ldots, K_{n-2} ; d u\right),
\end{aligned}
$$

where we used [11, (5.68)]. Hence we obtain that

$$
S\left(K_{1}, \ldots, K_{n-2},\left[0, e_{n}\right] ; \eta\right)=\frac{1}{n-1} S^{E}\left(K_{1}, \ldots, K_{n-2} ; \eta \cap E\right) .
$$

In order to prove that

$$
S\left(K_{1}, \ldots, K_{n-2}, B ; \eta\right)=V^{E}\left(K_{1}, \ldots, K_{n-2}, B\right)\left(\delta_{e_{n}}+\delta_{-e_{n}}\right)(\eta)
$$

we observe that for a convex body $K \subset E$, it is known (see [11, p. 220-221]) that

$$
S(K[n-1] ; \cdot)=\left(\delta_{u}(\cdot)+\delta_{-u}(\cdot)\right) V^{E}(K) .
$$


Considering $K=\sum_{i=1}^{n-2} \alpha_{i} K_{i}+\alpha_{n-1} B$, using the multilinearity of area measures and mixed volumes, and then comparing corresponding coefficients of both expressions, we obtain

$$
S\left(K_{1}, \ldots, K_{n-2}, B ; \eta\right)=V^{E}\left(K_{1}, \ldots, K_{n-2}, B\right)\left(\delta_{e_{n}}+\delta_{-e_{n}}\right)(\eta)
$$

which finishes the proof.

Proof of Theorem 1.9 We proceed by induction on $n \geq 3$ with $2 \leq i \leq n-1$. The first step of the induction is the case $n=3$, and hence $i=2$. More generally, for $n=i+1 \geq 3$, we know from [3, Theorem 1.1] that the assumption implies that $f$ is the support function of a convex body. Notice that in this case the integration defining the functional $\mathbf{F}$ involves the usual surface area measure and there are no other convex bodies.

Now we assume that the result is true for all $(n-1)$-dimensional Euclidean subspaces of $\mathbb{R}^{n}$ and $2 \leq i \leq n-2$. We prove that inequality (8) for the functional (6) defined on $\mathcal{K}^{n}$ and with $i \in\{2, \ldots, n-1\}$ implies that $f$ is a support function. Since the case $i=n-1$ is already covered by [3], Theorem 1.1], we can assume that $2 \leq i \leq n-2$. For this, let $f \in C\left(\mathbb{S}^{n-1}\right)$ be such that the functional $\mathbf{F}$ given in (6) satisfies (8), for all $K_{1}, \ldots, K_{n-i-1} \in \mathcal{K}^{n}$.

Let $E$ be any $(n-1)$-dimensional subspace of $\mathbb{R}^{n}$. Without loss of generality we can choose $E=\left\{x \in \mathbb{R}^{n}:\left\langle x, e_{n}\right\rangle=0\right\}=: e_{n}^{\perp}$ and identify it with $\mathbb{R}^{n-1}$. Let $B=B^{n} \cap E$ and $R \in \mathbb{R}$, $R>0$. For $\bar{K}, \bar{K}_{1}, \ldots, \bar{K}_{n-i-2} \in \mathcal{K}^{n-1}$ (arbitrary) define $\bar{F}: \mathcal{K}^{n-1} \longrightarrow \mathbb{R}$ by

$$
\overline{\mathbf{F}}(\bar{K})=\int_{\mathbb{S}^{n-1}} f(x) S\left(\bar{K}[i], \bar{K}_{1}, \ldots, \bar{K}_{n-i-2}, B+R\left[0, e_{n}\right] ; d x\right)
$$

We notice that as $2 \leq i \leq n-2$, we have $n \geq 4$.

From the assumption (8) on $\mathbf{F}$, it follows that $\overline{\mathbf{F}}$ satisfies

$$
\overline{\mathbf{F}}((1-t) \bar{K}+t \bar{L}) \geq \min \{\overline{\mathbf{F}}(\bar{K}), \overline{\mathbf{F}}(\bar{L})\}
$$

for all $t \in[0,1], \bar{K}, \bar{L} \in \mathcal{K}^{n-1}$, and any choice of $\bar{K}_{1}, \ldots, \bar{K}_{n-i-2} \in \mathcal{K}^{n-1}$. Lemma 5.1 shows that

$$
\begin{aligned}
\overline{\mathbf{F}}(\bar{K})= & \left.\frac{R}{n-1} \int_{\mathbb{S}^{n-2}} f\right|_{E}(x) S^{E}\left(\bar{K}[i], \bar{K}_{1}, \ldots, \bar{K}_{n-i-2} ; d x\right) \\
& +\left(f\left(e_{n}\right)+f\left(-e_{n}\right)\right) V^{E}\left(\bar{K}[i], \bar{K}_{1}, \ldots, \bar{K}_{n-i-2}, B\right)
\end{aligned}
$$


and similarly for $\bar{L}$ and $(1-t) \bar{K}+t \bar{L}$. We plug this into (35) and divide the resulting inequality by $R$. Then, for all $\bar{K}, \bar{L} \in \mathcal{K}^{n-1}$ and $t \in[0,1]$, we get

$$
\begin{aligned}
& \frac{1}{R} \overline{\mathbf{F}}((1-t) \bar{K}+t \bar{L})\left.\frac{1}{n-1} \int_{\mathbb{S}^{n-2}} f\right|_{E}(x) S^{E}\left(((1-t) \bar{K}+t \bar{L})[i], \bar{K}_{1}, \ldots, \bar{K}_{n-i-2} ; d x\right) \\
&+\frac{1}{R}\left(f\left(e_{n}\right)+f\left(-e_{n}\right)\right) V^{E}\left(((1-t) \bar{K}+t \bar{L})[i], \bar{K}_{1}, \ldots, \bar{K}_{n-i-2}, B\right) \\
& \geq \min \left\{\left.\frac{1}{n-1} \int_{\mathbb{S}^{n-2}} f\right|_{E}(x) S^{E}\left(\bar{K}[i], \bar{K}_{1}, \ldots, \bar{K}_{n-i-2} ; d x\right)\right. \\
& \quad+\frac{1}{R}\left(f\left(e_{n}\right)+f\left(-e_{n}\right)\right) V^{E}\left(\bar{K}[i], \bar{K}_{1}, \ldots, \bar{K}_{n-i-2}, B\right) \\
&\left.\frac{1}{n-1} \int_{\mathbb{S}^{n-2}} f\right|_{E}(x) S^{E}\left(\bar{L}[i], \bar{K}_{1}, \ldots, \bar{K}_{n-i-2} ; d x\right) \\
&\left.\quad+\frac{1}{R}\left(f\left(e_{n}\right)+f\left(-e_{n}\right)\right) V^{E}\left(\bar{L}[i], \bar{K}_{1}, \ldots, \bar{K}_{n-i-2}, B\right)\right\}
\end{aligned}
$$

When $R$ tends to infinity, we obtain that the functional defined on $\mathcal{K}^{n-1}$ and given by

$$
\left.\bar{K} \mapsto \int_{\mathbb{S}^{n-2}} f\right|_{E}(x) S^{E}\left(\bar{K}[i], \bar{K}_{1}, \ldots, \bar{K}_{n-i-2} ; d x\right)
$$

satisfies (8). Hence, the induction hypothesis yields that $\left.f\right|_{E}$ is a convex function in $E$. Since the same argument works for an arbitrary subspace $E$, we conclude that $f$ is a convex function, that is, (the homogeneous extension of) $f$ is the support function of a convex body.

\section{REFERENCES}

[1] S. T. Cheng, S. T. YAu, On the regularity of solutions of the $n$-dimensional Minkowski problem, Comm. Pure Appl. Math. 29 (1976), 495-516.

[2] A. Colesanti, Brunn-Minkowski inequalities for variational functionals and related problems. Adv. Math. 194 (2005), 105-140.

[3] A. Colesanti, D. Hug, E. Saorín Gómez, A characterization of some mixed volumes via the BrunnMinkowski inequality, J Geom. Anal. 24 (2014), 1064-1091.

[4] A. Colesanti, E. Saorín Gómez, Functional inequalities derived from Brunn-Minkowski inequalities for quermassintegrals, J. Convex Anal. 17 (2010), 35-49.

[5] R. J. GARdner, The Brunn-Minkowski inequality, Bull. Amer. Math. Soc. 39 (2002), 355-405.

[6] H. Hadwiger, Vorlesungen über Inhalt, Oberfläche und Isoperimetrie. Springer-Verlag, Berlin-GöttingenHeidelberg, 1957.

[7] P. MCMullen, Continuous translation invariant valuations on the space of compact convex sets, Arch. Math. 34 (1980), 377-384.

[8] P. MCMullen, Monotone translation invariant valuations on convex bodies, Arch. Math. 55 (1990), $595-598$.

[9] R. C. REILly, On the Hessian of a function and the curvatures of its graph, Michigan Math. J. 20 (1973), 373-383.

[10] P. SALANI, PhD Dissertation Thesis, University of Florence, 1997.

[11] R. SCHNEIDER, Convex Bodies: The Brunn-Minkowski Theory (second extended edition), Cambridge University Press, Cambridge, 2014. 
Dipartimento di Matematica e Informatica “U. Dini”, Viale Morgagni 67/A, 50134 Firenze, ITALY

E-mail address: colesant@math.unifi.it

KARlsruhe Institute of TeChnology (KIT), Department of MAThematics, D-76128 KarlsRuhe, GERMANY

E-mail address: daniel.hug@kit.edu

FAKULTÄT FÜR MATHEMATIK, OTTO-VON-GUERICKE UNIVERSITÄT MAGDEBURG, UNIVERSITÄTSPLATZ 2, D-39106 MAGDEBURG, GERMANY

E-mail address: eugenia.saorin@ovgu.de 